\title{
MINDFULNESS IN HEALTHCARE PROFESSIONALS AND MEDICAL EDUCATION
}

\author{
JACEK CHMIELEWSKI ${ }^{1}$, KACPER ŁOŚ2 ${ }^{2}$, and WŁODZIMIERZ ŁUCZYŃSKI ${ }^{3}$ \\ Medical University of Białystok, Białystok, Poland \\ ${ }^{1}$ Department of Psychiatry \\ ${ }^{2}$ Students' Scientific Section at the Department of Medical Simulations \\ ${ }^{3}$ Department of Medical Simulations
}

\begin{abstract}
Healthcare professionals are exposed not only to the ubiquitous stress, but also to the culture of perfectionism. Therefore, they need tools to achieve a balance between work and rest in order to effectively help their patients. The study objective is a review of the literature on the implementation of mindfulness in healthcare professionals and medical students. The authors searched the literature in PubMed and Google Scholar databases for publications about "mindfulness" in "healthcare professionals" and "medical students." The search included manuscripts published to July 31 , 2019. Mindfulness is a process of intentional paying attention to experiencing the present moment with curiosity, openness and acceptance of each experience without judgment. Mindfulness training leads to a better mood perception, lower stress perception, and responding to stimuli more effectively. All these features can have a potentially positive effect on healthcare service. The paper describes methods of intervention as well as their effects, which may be useful both in maintaining the well-being of healthcare professionals and in patient care. Mindfulness meditation has a beneficial effect on stress, depression, burnout, well-being and empathy among doctors, nurses and other healthcare workers. However, the method has a number of limitations, including a small number of participants, a high dropout rate in the intervention group and, above all, ceasing to practice mindfulness in the longer term after the course termination. Mindfulness can be widely implemented by healthcare professionals, thus improving their well-being and the quality of care they provide. Further standard scientific research is needed to confirm this impact. Int J Occup Med Environ Health. 2021;34(1):1-14
\end{abstract}

Key words:

mindfulness, medical education, healthcare, medical students, nurses, doctors

\section{INTRODUCTION}

Healthcare professionals, on the one hand, face a number of stressors in the medical environment, including those always existing in medicine (long work hours, night shifts, pain and suffering of patients, caring for patients with lifethreatening diseases, etc.) and the ones that have emerged recently (staffing shortages, increased numbers of complex patients, diverse forms of healthcare financing, dynamic changes in technology and regulations, etc.). There are certainly many reasons standing behind: some are due to the lack of a proper work structure and education, others involve the shortage of human resources and materials, as well as insufficient remuneration; high patient demand also exerts an impact. Constant stress experienced by healthcare professionals can lead to burnout syndrome, attrition, depression and aggressive behaviors [1,2]. They show a very high level of emotional exhaustion and depersonalization [3]. Burnout syndrome and reduced quality of

Funding: this study was supported by the Medical University of Bialystok, Poland (grant No. N/ST/ZB/18/001/1206 entitled "Mindfulness and executive functions in medical students during high-fidelity simulations," chief investigator: Włodzimierz Łuczyński).

Received: September 14, 2019. Accepted: July 27, 2020.

Corresponding author: Włodzimierz Łuczyński, Medical University of Białystok, Department of Medical Simulations, Szpitalna 30, 15-029 Białystok, Poland (e-mail: wlodzimierz.luczynski@umb.edu.pl). 
life among doctors and nurses undoubtedly have a negative impact on well-being, performance and patient care. The mechanisms of the above correlations have not been fully elucidated yet.

On the other hand, the modern work environment of physicians rewards efficiency, speed and productivity both among general practitioners (GPs) and specialists. These requirements force, in part, overlearned and automatic actions in response to frequently recurring cases, symptoms and medical conditions. However, these automatisms can be dangerously mindless, leading to stereotypies, monotony, inefficiency and a reduced quality of the medical services provided. Similar mechanisms may also apply to patients. In medical culture, too little attention has been paid to the well-being of medical students and healthcare workers. Despite the growing awareness that self-care and self-awareness are key competences of healthcare professionals, these issues seem to be largely neglected in the process of medical education. Self-acceptance is the foundation of any kind of care for another human being [4]. Since the features of mindfulness and self-compassion should be considered to be at least partially modifiable, more attention is required to shape them both in students and young as well as experienced doctors.

For some time now, attempts have been made to prevent burnout syndrome through individual and systemic approaches. Both the limitation and organization of work, as well as mind/body training can bring benefits in this field. General recommendations for doctors, nurses and students on this issue have to be outlined [5]. The outcomes of various types of interventions in this area are frequently inconsistent, and no research has been done on the impact of burnout in premedical students, or on suicide prevention among doctors or nurses [6]. Further long-term, prospective and randomized studies are necessary. Perhaps interventions at a relatively low cost, both time-efficient and collegial, will improve the quality of life, reduce oc- cupational burnout, and eventually provide better healthcare service to the society.

Thus, healthcare workers are not only exposed to the pervasive stress associated with the amount of work, study and activity, but also to the culture of perfectionism that surrounds them all. They need tools to take a step back, see their actions from perspective, achieve a balance between work, study and rest, and set priorities to both maintain mental health and well-being, and deliver effective healthcare.

\section{METHODS}

The study objective is a narrative review of the literature with support commentary on the implementation of mindfulness in healthcare professionals and medical students. The authors aimed to include doctors, nurses and medical students in a concise review, and add some personal experience in the field of medical education and mindfulness meditation. They searched the literature in PubMed and Google Scholar databases for publications about "mindfulness" in "healthcare professionals" and "medical students." The search included manuscripts published to July 31, 2019. They described intervention methods and their effects, which may be useful in maintaining the wellbeing of healthcare professionals and in patient care. Limitations and difficulties in implementing mindfulness in healthcare service were also provided. Further directions for the development of research in this field of knowledge were proposed. They also added some personal experience comments in the field.

\section{RESULTS}

\section{Mindfulness as a stress reducing tool}

Mindfulness is a process of intentionally paying attention to each moment with curiosity, openness and acceptance of each experience without judgment [7]. This is achieved through an attitude of acceptance, clarity, a lack of valuation, patience, sincerity, unexpectedness, loving kind- 
ness, care and compassion for the current situation. This experience allows switching from the normal mode of an automatic reaction to a more careful response that may lead to a better understanding of oneself and the surrounding world. The mindfulness technique helps avoid the "autopilot" effect that may eventually cause unwanted rumination, i.e., repeated negative looping thoughts about oneself in the past and future. Mindfulness features correlates with lower levels of stress, depression and anxiety, while mindfulness training leads to a better mood perception, the implementation of coping strategies for stress, the ability to perceive a given situation more clearly, and responding to stimuli more effectively [8]. All these features can have a potentially positive effect on workplace functioning in healthcare and medical education.

Implementing mindfulness in medicine and psychotherapy has accelerated considerably in recent years. In the USA, mindfulness training is used in $>250$ medical centers. The classic Mindfulness-Based Stress Reduction (MBSR) program in adults who struggle with stress and its health consequences yields some improvement in well-being, as well as reduces stress and its symptoms [9]. The loving kindness meditation, which involves developing unconditional kindness to everyone, is a major mindfulness component [10]. This skill can be extremely useful in work with patients and in medical team work.

All the features of stress management described above can be implemented in medical and nursing practice. It can be assumed that support based on cognitive, behavioral and mindfulness approaches will help reduce stress of doctors, nurses and medical students, ease anxiety and the feeling of burnout, and improve patient care [11].

\section{Medical staff}

Doctors

Mindfulness can become the missing link between evidence-based medicine and relationship-centered care. Perhaps the routine diagnostic and therapeutic process that does not always produce a desired effect should be enriched with the awareness of the inhibitors and facilitators of rationality in decision making. Learning critical thinking, developing cognitive and affective biases, and using such processes as reflection and mindfulness can lead to greater creativity, lateral thinking, and innovation in the diagnosis and treatment of patients [12]. Scientific research in this field focuses both on the relationship of mindfulness traits with the quality of life of physicians and their approach to patients, as well as on possible changes in this area. Research accomplished as an intervention can be of quantitative or qualitative nature, and is carried out as short- or long-term observations. Their results are generally consistent, but often differ in details. There are very few randomized and long-term studies in this field.

In the observation of 12 young doctors (average age: 27.6 years), the features of mindfulness and self-compassion correlated positively with providing calm, compassionate care, resilience and clinician's mental health [13]. In contrast, chronic stress showed a strong inverse correlation with these characteristics. This proves that mindfulness and self-compassion deeply affect the traits that medical educators seek in their students and future doctors: clinician's well-being and confidence in providing calm, compassionate care. According to the authors of that report, it remains to determine which type of training most effectively improves mindfulness and self-compassion among physicians. The limitation of this observation was not only a small number of participants, but also voluntary self-enrollment in the elective meditation-focused seminar.

In turn, mind-body medicine (MBM) facilitators from the Georgetown University School of Medicine were characterized by lower levels of stress (assessed according to the Perceived Stress Scale) and a higher level of mindfulness (according to the Freiburg Mindfulness Inventory) in comparison with normative controls [14]. These effects were also shown on the hormonal level including salivary cortisol, 
dehydroepiandrosterone sulfate, testosterone and secretory immunoglobulin A [15]. With the above in mind, the Mentoring and Professionalism in Training Program for pathology residents, in addition to other themes, takes mindfulness into account as an important issue in the promotion of humanism in healthcare professionals in the health system [16].

Conducting a full 8-week MBSR course for doctors working in a hospital or university seems to be a timeconsuming and expensive task. Therefore, simpler and equally effective solutions are searched for. An interesting experiment was carried out at the University of Wisconsin, in which leaders were prepared and trained to promote the mindfulness concept among healthcare providers [17]. The effects exceeded the initiators' expectations and the investment that had been made. Five leaders from primary care departments not only appreciated the value of mindfulness but, above all, fostered mindfulness activities among their colleagues, students and patients in the state of Wisconsin and beyond. Indeed, it appears to be an excellent idea to spread the concept of mindfulness in the medical world at little expense. Obviously, this type of a project has its limitations, including the sample size, the lack of statistical analysis, the lack of measurements before and after the intervention, and no randomization. In a similar program conducted by a colleague experienced in mindfulness training, pediatric residents experienced improvements in their positive attitude, perceived stress and resilience after mind-body skills training even though they completed, on average, 4.3 out of 8 online modules, and 2.8 of 4 personal sessions [18]. The longterm effects (after 6 months) in that study included a reduction in the symptoms of burnout and an increase in mindfulness. The course participants indicated that the facilitator helped them introduce new skills to the residents' lives. However, no changes were noted in the field of selfcompassion. Perhaps this was due to the lack of formal, standardized education of the course leader.
This approach has its strengths and weaknesses. The major problems of this type of research is unwillingness to participate in such programs, self-selection, motivation, etc. Additionally, exam sessions or their completion during or after the course may be a disruptive factor (stress and mood improvement or worsening regardless of the course being held). The lack of course continuation as part of the proposed post-course maintenance sessions is a major concern. Only 2 residents in the first 2 sessions took part in the maintenance session in that study. Unfortunately, this can be observed in all mindfulness-based courses. Very few people decide to enroll in the post-course sessions or come to the subsequent group meetings (the authors' own experience based on work with MBSR groups). The continuation of the mindfulness practice is one of the major issues and challenges in this field, especially in extremely busy healthcare professionals.

Another idea to achieve an effect using smaller resources is to shorten the full MBSR course. In an uncontrolled pilot study, GPs were subjected to the abbreviated version of MBSR [3] and, as a result, the features of burnout, depression, anxiety and stress were found to be reduced both immediately after the intervention and 9 months later. Interestingly, also in this case, no changes were observed in resilience and compassion. Satisfactory attempts were also made to reduce the costs of the MBSR program by replacing 6 out of 8 traditional personal meetings by telephone group sessions (tMBSR) [19]. The impact of the course was greater in people who continued their mindfulness practice after completing the course as compared to those who did not. This indicates that the program can be implemented in groups of healthcare professionals who are not available in a traditional, personal way (on-site programs).

In one of the first randomized trials, MBSR was used among 148 residents of medicine, surgery and primary care [20]. In the whole group, 3 months after the baseline assessment, no significant differences were noted between 
the group subjected to mindfulness and the wait-list control group in terms of emotional exhaustion. However, greater improvements were observed after MBSR with regard to personal accomplishment, worry, mindfulness skills, selfcompassion and perspective-taking. In the further analysis of this material, it was pointed out that persons with greater baseline emotional exhaustion did seem to benefit from the MBSR course. In another report, it was stated that stress reduction after MBSR in healthcare professionals depended on the reduction in rumination and negative affect [21]. This effect was maintained throughout the observation period 3 months after the course. A study conducted in the Galveston University medical staff revealed that the effect of MBSR in terms of perceived stress and well-being persisted and was stable over the year of a follow-up after the intervention [22]. In another observation, primary care physicians participating in a full MBSR course reported a significant decrease in depersonalization, and an increase in dedication and mindfulness skills. Changes in empathy were not observed [23].

Few data are available on the impact of mindfulness on the representatives of particular medical specialties. In a very small group of psychiatry residents (11 people), empathy increased, and perceived stress decreased, after a mindfulness course, although this last change was not statistically significant [24].

It is extremely difficult to check whether mindfulness affects the quality of patient care, as it requires randomized, long-term clinical trials. The mindfulness course specially designed for healthcare professionals resulted in stress, anxiety and burnout reduction [25]. As a qualitative effect, a positive impact was reported on the quality of patient care and on planning to use this technique both in personal development and in work with patients. Both teaching and assessing the professionalism of young doctors and residents appear a great challenge. The MBSR courses may be helpful - after completing them, residents admitted increased awareness, self-reflection and self-ac- ceptance at work, and - importantly for their professionalism and patient care - acceptance for their own limitations [26]. Moreover, they mentioned being more resilient, and better at setting priorities and limits. In addition, the residents asked for help more often and seemed to be more open towards feedback. Importantly, they indicated an enhanced sense of compassion for others. Physicians who completed a 52-hour program focusing on mindful communication experienced its 3 aspects:

- sharing experiences from medical practice reduced the sense of isolation;

- mindfulness skills improved the ability to focus attention and boosted attentive listening with adequate response;

- development of self-awareness was a positive experience, although the participants did not always give themselves permission to attend to their own development [27].

A very interesting observation was made when searching for the relationship between mindfulness, the quality of life and the quality of patient care (from the doctor's perspective) among American oncologists [28]. In this group of doctors, mindfulness influenced life satisfaction and a less suboptimal patient care. Mindfulness moderated the relationship between the oncologists' sleep problems and suboptimal patient care. It is likely that the skills acquired during mindfulness courses will be passed on to patients, especially those with chronic, psychosomatic or life-threatening diseases. Undoubtedly, the above data show a positive effect of mindfulness on the mental health of doctors.

Nurses

Mindfulness meditation has a beneficial effect on stress, anxiety, depression, burnout, well-being and empathy among nurses. However, most reports refer to a small number of respondents and a short observation time, thus limiting the generalization of results. In 13 Brazilian hos- 
pital nurses, a 6-week Stress Reduction Program (based on MBSR by Kabat-Zinn), with elements of mindfulness and loving kindness meditation, reduced the symptoms of stress, burnout, depression and anxiety (as a trait), with the effects sustained throughout a long-term followup [29]. The course participants admitted experiencing such feelings as: a more attentive perception of internal and external experience, a greater attention and awareness of actions and attitudes at every moment, and finally a positive influence on nursing activities. The limitations of the study included a small number of subjects, no control group and no randomization. The strength of the study was certainly a positive effect persisting after the end of the course.

The latest study, being one of the first fully randomized, standardized studies in this field, investigated the impact of an 8-week classic MBSR course on 110 nurses working in general hospitals [30]. After the intervention, stress and negative affect decreased, whereas positive affect and resilience increased. As for job satisfaction, the positive trend did not reach statistical significance. Among the challenges of the MBSR programs for nurses, restlessness, physical pain and dealing with difficult emotions are mentioned [31]. Some positive effects of the mindfulness course, such as an increase in individual and workplace resilience, were also observed among midwives [32].

In a study covering a broad spectrum of healthcare providers, including nurses, doctors, psychologists and social workers, a positive impact on emotional exhaustion, depersonalization and personal accomplishment was demonstrated [33]. Various groups of healthcare professionals are interested in mindfulness courses: nurses, physicians, social workers, dietitians, psychologists and others [34]. The most popular topics that healthcare workers would like to explore are "Introduction to stress, resilience and relaxation response" and "Autogenic training." Half of those willing to participate usually complete at least 1 module, but only $9 \%$ take part in all 12 modules. Nearly all (90\%) plan to use this technique for themselves and in work with the patient. Summing up, mindfulness meditation eases work-related stress among nurses.

\section{Medical students}

Medical universities are constantly raising the level of education and requirements for both prospective undergraduate students and graduates. Standardized examinations and new subjects are introduced, and the number of learning hours increases. It may thus seem that the level of professionalism among young doctors is also higher. Paradoxically, however, students report being overloaded with activities, and burdened with constant stress and a sense of competition. According to some research, medical students also show low emotional and cognitive empathy scores, and an avoidant attachment style [35]. This may increase burnout and decrease the ability to connect with patients [36].

Despite the awareness that medical studies are very stressful, and that stress has an impact on mental and physical health, no effective methods have been established to combine intensive learning, interest in research, and the ability to take self-care and improve mentally. Older physicians begin to be interested in building relationships with patients, which they enjoy. However, this type of an attitude could be adopted by students during their medical education, yet it requires a change in thinking and in the approach to studying medicine, along with a conscious willingness to build a relationship with the patient and to learn how to do it. This path may lead to the concept of relationship-centered care, self-awareness and mindfulness as the basis of medical education.

Medical universities, apart from accreditation organizations, should develop programs to support mental health and well-being of their graduates. Healthcare professionals are co-responsible for the health and well-being of those they serve. Therefore, strategies are needed to help both students and workers to maintain resilience and well- 
being in their professional and personal lives so that they could provide service to the best of their knowledge and skills for as long as possible. Such strategies are already being introduced, mostly in a pilot or facultative form, but not only. Examples include programs implemented in U.S. universities, such as Resilience and Well-being for Health Professionals, Mind-Body Medicine Skills, and Mindful Resilience [37]. Their long-term follow-up outcome will be crucial.

Self-awareness and reflection have long been promoted through mind-body skills among medical students [38]. However, the study results are still insufficient, do not refer to larger sample groups, do not assess long-term effects, and do not investigate the relationship with learning and further work as a doctor. Perhaps mindfulness will extend the teacher-student relationship for the benefit of both, and emphasize the healer's role in medicine [39]. Undoubtedly, being a good clinical teacher and being a mindful teacher share many characteristics.

Such mindfulness effects as enhanced empathy, reduced anxiety and stress, and increased self-regulation (the ability to effectively manage one's thoughts and actions to complete a task) can be particularly useful for medical students who struggle with heavy workload, have to memorize a lot of information in a short time, and are constantly subjected to tests. Mindfulness courses can lead to a greater sense and resilience among medical students, an ability to manage their workflow better, and more acceptance of their limitations as learners [40]. A meta-analysis of 19 studies concerning the use of mindfulness among medical students has shown that mindfulness-based interventions decrease stress, anxiety and depression, as well as improve mindfulness, mood, self-empathy and empathy [41]. As revealed by the authors of that analysis, mindfulness training can be relatively easily adapted to and integrated with the modern teaching of medicine.

The vast majority of mindfulness classes are based on voluntary student participation. In a very interesting study conducted at Boston University, the Embodied Health Elective was carried out combining yoga, mindfulness techniques and educational elements oriented towards scientific research into mind-body practices, in a group of 27 first- and second-year medical students [42]. As a result, a significant increase was observed in self-regulation and self-compassion. In addition, there was an improvement tendency in terms of empathy and perceived stress, but these effects did not reach statistical significance. According to the participating students, these changes were caused by:

- reconnection between the body and mind,

- community in a competitive environment,

- increased mindfulness,

- confidence in the use of mind-body skills with patients, - stress management.

The limitations of the study were: no control group, selfenrollment in the study, a small number of students, a short observation time, and a fairly high empathy score at baseline, suggesting a ceiling effect for this measure. Similarly, MBSR reduced tension, anxiety and total mood disturbance in second-year medical students as compared to those who participated in seminars on complementary medicine [43]. A similar course based on meditation reduced stress and increased empathy in premedical and medical students [44]. Students of medicine and psychology experienced a change towards reduced reactivity, increased curiosity, affect tolerance, patience and selfacceptance, and improved relational qualities [45]. Certainly, mindfulness meditation reduces the level of cortisol in the serum of medical students [46].

Stress and depression have a negative impact on empathy. Thus, mindfulness seems to be a good instrument to improve empathy and the quality of patient care. However, not all studies seem to confirm the impact of mindfulness on reducing stress and improving empathy. For example, in a study conducted among first-year students of medicine, an increase in stress and a decrease in empathy 
were observed regardless of the MBM training. However, the completion of this course protected them from an increase in depression, which was observed in the group without therapy [47]. Perhaps the observed stress was caused by heavy workload and credits. In another study, nursing students after MBSR intervention showed an increase in mindfulness, but no effect on stress or the quality of life was noted [48].

The timing of such interventions seems to be crucial. An MBSR course carried out during the first year of medical studies showed a small-to-moderate influence on mental health and no impact on the students' empathy in a 20-month observation period [49]. In the authors' subjective opinion, the decisive factor may be experiencing lower stress during clerkships as compared to full-time studying with credits, tests and exams, as is the case among residents. In another study, it was clearly shown in a small group of graduate healthcare students that statistically significant effects of MBSR concerning burnout and empathy can be brief if not sustained by regular practice [50].

Some studies have attempted to qualitatively describe the changes obtained through mindfulness intervention. The students of medicine and nursing who underwent the mind-body skills course identified 3 main topics of their interest in this aspect: "ability to be more present," "increased perception and awareness of oneself" and "connection on a deeper level with others" [51]. All these features can be very useful in their future work with patients. Gender differences may exist in the mindfulness reception. In a study conducted after an MBSR course, an improvement was noted only in female medical students in terms of mental distress, study stress, subjective wellbeing and mindfulness [52]. It might be due to the high attendance and performance of all recommended tasks by female students. Further research conducted in this group demonstrated that higher neuroticism and conscientiousness, more frequently found in female students, predispose them to better effects in terms of mental distress and subjective well-being [53]. Thus, female students seem to be an important target group of mindfulness intervention. In another study, it was also shown that the medical students interested in mindfulness courses are characterized by higher psychological distress, neuroticism, worrying, problem avoidance and lower mindfulness skills compared to those unwilling to do the course [49].

Interestingly, after graduating, the choice of residency in the field of primary care practice among 208 osteopathic medical students was not related to mindfulness assessed using the Five Facet Mindfulness Questionnaire [54]. On the other hand, the students who chose emergency medicine as their future specialization and participated in a mindfulness course during summer holiday clerkships noted some changes in self-reported behaviors and attitudes that lasted for $\leq 6$ months after intervention [55]. Despite the small size of the group sample (20 students), this may indicate that burnout syndrome can be prevented in future emergency medicine physicians.

In all the studies described above, students enrolled themselves voluntarily in mindfulness programs during their university education. In the only known study that assessed the obligatory course of stress management and resilience, introduced in the first year of medicine, no positive intervention effect was observed as compared to the control cohort [56]. The mental quality of life, happiness decline and stress scores were all statistically significantly increased in both cohorts during the observation year. No differences were noted in burnout or resilience. Perhaps people who voluntarily participate in mindfulness courses are the ones who benefit the most from them.

Another limitation of this type of research may be the comparison of mindfulness interventions with the wait-list control group not receiving any support during the observation period. The German MediMind study compared the effect of mindfulness therapy with standard treatment (autogenic training) and a control group without intervention [57]. No statistically significant positive effect of 
mindfulness therapy on stress management among medical students was observed due to, among other things, high dropout rates for the students who suffered from psychological symptoms. However, the authors of that report pointed out that the number of people willing to participate was high (183 people), which indicates the need for further development of activities in this direction.

In the only known long-term follow-up (6 years) involving 288 Norwegian students of medicine and psychology, positive effects were demonstrated in terms of wellbeing, problem-focused coping and avoidance-focused coping [58]. These effects were noted despite the relatively low levels of adherence to formal mindfulness practice, which suggests that mindfulness acts not as a state, but as a trait, and it indicates the importance of informal practices in shaping mindfulness attitudes on a daily basis. Medical school students in Malaysia assessed that the mindfulness-based stress management program (MBSM/MindfulGym) could be useful in helping patients [59]. Mindfulness not only reduces stress in medical students, but also helps them look forward more confidently and hopefully to becoming physicians [60].

\section{Mindfulness-related problems and development perspectives}

The analysis of the current study stimulates a number of questions and raises doubts as to the application of mindfulness in healthcare workers. Numerous authors have concluded that conducting an MBSR course is feasible among healthcare professionals and students. The critical analysis of a great deal of studies seems to indicate that many circumstances interfere with the task. A small number of course participants, a high course withdrawal rate and, first of all, course discontinuation or neglecting mindfulness practice in the longer term after the course termination, prove that, as stated by Kabat-Zinn [7], being mindful in everyday life is simple but not easy. The authors of this article share this experience - the number of participants was small and the course was completed by almost everyone; however, in the subsequent 1-2 years, not more than several percent of those interested continued their weekly practice (personal data, unpublished). In the authors' opinion, maintaining the achieved attitude in further personal and professional life will be critical. Therefore, all the barriers that limit regular practice and meditation after mindfulness training have to be identified, and specific tools have to be used to remind the participants of mindfulness practice. One can only assume that those who, thanks to mindfulness, can cope with stress effectively will continue the practice. It should also be taken into account that some students or healthcare workers are already at the adequate level of mindfulness. As shown by the above research survey, a mindfulness course is easier to conduct among students than among doctors or nurses, as the former are more available than professionally active employees. However, the outcome of the research conducted on students cannot fully translate into the real work of physicians, since the final effect involves a great deal of additional factors, as well as professional environment or family, etc., which determine the attainment of mindfulness objectives in everyday work with patients in hospital or outpatient settings. In addition, no one will appreciate the impact of mindfulness on well-being, communication with patients and effective work/rest like the highly experienced healthcare workers. A higher level of stress in medical students or doctors as compared to general population can be another factor influencing the effectiveness of mindfulness.

Long-term, randomized studies assessing the impact of mindfulness on burnout and the quality of patient care are constantly lacking. The lack of a control group is one of the major problems. Voluntary enrollment in the intervention hinders the outcome translation into the whole healthcare population. Likewise, it is not known how the people who continue mindfulness practice differ from those who do not. Undoubtedly, such factors as the type 
of mindfulness practice, its organization mode, implementation in everyday activities, duration, and optional or obligatory participation in the course will exert an impact on the outcome. Being aware of the realities of work in medicine, the authors realize that it is almost impossible to conduct a randomized study with a long-term follow-up in a large group.

There is a growing interest in the issue of the impact of mindfulness on teaching effects and effective learning, which refers both to students and young as well as experienced doctors. However, no research data are available.

The question arises whether the student's condition before university education has an effect on the development of stress and its complications. Can doctors be taught to cope not only with their own stress but also with their patients' stress? Mindfulness interventions aim to create a possibility for being more aware of one's own experience, and for a better recognition of stressors, and regulations of thoughts and feelings, in order to block harmful effects. The objective of mindfulness is to allow healthcare workers to keep a distance from a mentally and emotionally stressful environment. The structure of medical universities should incorporate instruments and programs to accomplish these objectives. The questions that still remain open are as follows: when should mindfulness be taught on the path of a medical career? Which format is the best and who should it address? How can long-term effects of mindfulness be maintained? Should mindfulness be included in the university curriculum for medical students? Should mindfulness be taught as an elective or obligatory subject? It would be interesting to check when the interventions are optimal - at the beginning or end of the study, at the start or finish of the residency period?

Research into whether mindfulness traits influence diagnostic and therapeutic decisions in life-threatening conditions can be extremely interesting. There are more questions than answers in this field, and no studies are available. Does being here and now result in a better or worse doctor's performance at work? Does anyone being more mindful respond better to stressful situations in medicine? Is anyone who escapes rumination and observes his/her own feelings more effective in emergency medicine actions? Does mindfulness help in managing human teams in life-threatening events? Do the features of mindfulness help make quick and proper diagnostic and therapeutic decisions? Is mindfulness associated with technical or soft skills of students and doctors? Does being more mindful result in more attentive listening to patients and the accompanying persons or members of the medical team?

The authors tried to answer some of these questions by conducting research assessing the relationship of the features of mindfulness with technical and soft skills of medical students during high-fidelity medical simulations [61]. Their unpublished preliminary experiments and analyses seem to indicate that the mindfulness of a student who is in charge of a medical team in life-threatening events (medical simulation) is associated with soft skills and stress perception by the simulation participant, but it does not affect technical skills. These findings require further research and have to be confirmed in larger groups in standardized conditions.

Thus, the ideal assessment of mindfulness in healthcare professionals should:

- be a randomized study,

- eliminate the impact of voluntary enrollment in the intervention (if it is at all possible),

- be based on a standardized course of mindfulness (e.g., an 8-week MBSR course),

- be a long-term assessment,

- evaluate the effects for the student/doctor/nurse's wellbeing,

- evaluate the effects in theoretical and practical exams (if the impact on learning/training is examined),

- assess the impact of mindfulness on team work,

- assess the effects at work with patients (if possible),

- evaluate both quantitative and qualitative effects. 


\section{CONCLUSIONS}

In conclusion, the implementation of the well-being enhancing techniques in healthcare professionals and medical education should be recommended for the benefit of both patients and medical service providers. The mindful perspective may have an impact on therapeutic decisions and cooperation with patients, leading to better health effects. The analysis of the above data seems to suggest that enhanced self-awareness in healthcare professionals and medical students will allow them to more frequently engage in self-care activities and to manage stress more effectively. They can be also better equipped with instruments improving wellness in their patients. Mindfulness can be widely applied in healthcare professionals, boosting their well-being and improving the quality of care provided. However, further research conducted according to scientific standards is needed to confirm this correlation.

\section{REFERENCES}

1. Shanafelt TD, Boone S, Tan L, Dyrbye LN, Sotile W, Satele D, et al. Burnout and satisfaction with work-life balance among US physicians relative to the general US population. Arch Intern Med. 2012;172(18):1377-85, https://doi.org/10.1001/ archinternmed.2012.3199.

2. Botha E, Gwin T, Purpora C. The effectiveness of mindfulness based programs in reducing stress experienced by nurses in adult hospital settings: a systematic review of quantitative evidence protocol. JBI Database System Rev Implement Rep. 2015;13(10):21-9, https://doi.org/10.11124/jbisrir-2015-2380.

3. Fortney L, Luchterhand C, Zakletskaia L, Zgierska A, Rakel D. Abbreviated mindfulness intervention for job satisfaction, quality of life, and compassion in primary care clinicians: a pilot study. Ann Fam Med. 2013;11(5):412-20, https://doi. org/10.1370/afm.1511.

4. Schmidt S. Mindfulness and healing intention: concepts, practice, and research evaluation. J Altern Complement Med. 2004;10 Suppl 1:s7-14, https://doi.org/10.1089/1075553042 245917.
5. Ripp JA, Privitera MR, West CP, Leiter R, Logio L, Shapiro J, et al. Well-Being in Graduate Medical Education: A Call for Action. Acad Med. 2017;92(7):914-7, https://doi. org/10.1097/ACM.0000000000001735.

6. Williams D, Tricomi G, Gupta J, Janise A. Efficacy of burnout interventions in the medical education pipeline. Acad Psychiatry. 2015;39(1):47-54, https://doi.org/10.1007/s40596-014-0197-5.

7. Kabat-Zinn J. Mindfulness-Based Interventions in Context: Past, Present, and Future. Clin Psychol: Sci Pract. 2003;10(2):144-56, https://doi.org/10.1093/clipsy.bpg016.

8. Shapiro SL, Carlson LE, Astin JA, Freedman B. Mechanisms of mindfulness. Clin Psychol. 2006;62(3):373-86, https://doi. org/10.1002/jclp.20237.

9. Carmody J, Baer RA. Relationships between mindfulness practice and levels of mindfulness, medical and psychological symptoms and well-being in a mindfulness-based stress reduction program. J Behav Med. 2008;31(1):23-33, https:// doi.org/10.1007/s10865-007-9130-7.

10. Hofmann SG, Grossman P, Hinton DE. Loving-kindness and compassion meditation: potential for psychological interventions. Clin Psychol Rev. 2011;31(7):1126-32, https:// doi.org/10.1016/j.cpr.2011.07.003.

11. Regehr C, Glancy D, Pitts A, LeBlanc VR. Interventions to reduce the consequences of stress in physicians: a review and meta-analysis. J Nerv Ment Dis. 2014;202(5):353-9, https:// doi.org/10.1097/NMD.0000000000000130.

12. Croskerry P. Adaptive expertise in medical decision making. Med Teach. 2018;40(8):803-8, https://doi.org/10.1080/01421 59X.2018.1484898.

13. Olson K, Kemper KJ. Factors associated with well-being and confidence in providing compassionate care. J Evid Based Complementary Altern Med. 2014;19(4):292-6, https://doi. org/10.1177/2156587214539977.

14. Talisman N, Harazduk N, Rush C, Graves K, Haramati A. The impact of mind-body medicine facilitation on affirming and enhancing professional identity in health care professions faculty. Acad Med. 2015;90(6):780-4, https://doi.org/ 10.1097/ACM.0000000000000720. 
15. Maclaughlin BW, Wang D, Noone AM, Liu N, Harazduk N, Lumpkin M, et al. Stress biomarkers in medical students participating in a mind body medicine skills program. Evid Based Complement Alternat Med. 2011;2011:950461, https:// doi.org/10.1093/ecam/neq039.

16. Esposito MJ, Roychoudhury S, Fornari A. A Professionalism and Mentoring Curriculum for Pathology Residents in Training. Acad Pathol. 2018;5:2374289518805062, https:// doi.org/10.1177/2374289518805062.

17. Luchterhand C, Rakel D, Haq C, Grant L, Byars-Winston A, Tyska S, et al. Creating a Culture of Mindfulness in Medicine. WMJ. 2015;114(3):105-9.

18. Romcevich LE, Reed S, Flowers SR, Kemper KJ, Mahan JD. Mind-Body Skills Training for Resident Wellness: A Pilot Study of a Brief Mindfulness Intervention. J Med Educ Curric Dev. 2018;5:2382120518773061, https://doi.org/10.1177/ 2382120518773061.

19. Bazarko D, Cate RA, Azocar F, Kreitzer MJ. The Impact of an Innovative Mindfulness-Based Stress Reduction Program on the Health and Well-Being of Nurses Employed in a Corporate Setting. J Workplace Behav Health. 2013;28(2):10733, https://doi.org/10.1080/15555240.2013.779518.

20. Verweij H, van Ravesteijn H, van Hooff MLM, LagroJanssen ALM, Speckens AEM. Mindfulness-Based Stress Reduction for Residents: A Randomized Controlled Trial. J Gen Intern Med. 2018;33(4):429-36, https://doi.org/10.1007/ s11606-017-4249-x.

21. Martín-Asuero A, García-Banda G. The Mindfulness-based Stress Reduction program (MBSR) reduces stress-related psychological distress in healthcare professionals. Span J Psychol. 2010;13(2):897-905, https://doi.org/10.1017/s113874 1600002547.

22. Geary C, Rosenthal SL. Sustained impact of MBSR on stress, well-being, and daily spiritual experiences for 1 year in academic health care employees. J Altern Complement Med. 2011;17(10):939-44, https://doi.org/10.1089/acm.2010.0335.

23. Verweij H, Waumans RC, Smeijers D, Lucassen PL, Donders AR, van der Horst HE, et al. Mindfulness-based stress reduction for GPs: results of a controlled mixed methods pilot study in Dutch primary care. Br J Gen Pract. 2016; 66(643):e99-105, https://doi.org/10.3399/bjgp16X683497.

24. Hoenders HJ, Booij SH, Knegtering H, van den Brink H. [Mindfulness training for psychiatrists in residency: a pilot study]. Tijdschr Psychiatr. 2016;58(11):809-13. Dutch.

25. Kinser P, Braun S, Deeb G, Carrico C, Dow A. "Awareness is the first step": An interprofessional course on mindfulness and mindful-movement for healthcare professionals and students. Complement Ther Clin Pract. 2016;25:18-25, https:// doi.org/10.1016/j.ctcp.2016.08.003.

26. Verweij H, van Ravesteijn H, van Hooff MLM, Lagro-Janssen ALM, Speckens AEM. Does Mindfulness Training Enhance the Professional Development of Residents? A Qualitative Study. Acad Med. 2018;93(9):1335-40, https://doi. org/10.1097/ACM.0000000000002260.

27. Beckman HB, Wendland M, Mooney C, Krasner MS, Quill TE, Suchman AL, et al. The impact of a programin mindful communication on primary care physicians. Acad Med. 2012;87(6):815-9, https://doi.org/10.1097/ACM.0b013e3182 $53 \mathrm{~d} 3 \mathrm{~b} 2$.

28. Kracen A. Mindfulness, health, well-being, and patient care of oncologists. Richmond, Virginia: Virginia Commonwealth University; 2009.

29. Dos Santos TM, Kozasa EH, Carmagnani IS, Tanaka LH, Lacerda SS, Nogueira-Martins LA. Positive Effects of a Stress Reduction Program Based on Mindfulness Meditation in Brazilian Nursing Professionals: Qualitative and Quantitative Evaluation. Explore (NY). 2016;12(2):90-9, https://doi.org/ 10.1016/j.explore.2015.12.005.

30. Lin L, He G, Yan J, Gu C, Xie J. The Effects of a Modified Mindfulness-Based Stress Reduction Program for Nurses: A Randomized Controlled Trial. Workplace Health Saf. 2018;2165079918801633, https://doi.org/10.1177/216507991 8801633.

31. Cohen-Katz J, Wiley S, Capuano T, Baker DM, Deitrick L, Shapiro $\mathrm{S}$. The effects of mindfulness-based stress reduction on nurse stress and burnout: a qualitative and quantitative 
study, part III. Holist Nurs Pract. 2005;19(2):78-86, https:// doi.org/10.1097/00004650-200503000-00009.

32. Foureur M, Besley K, Burton G, Yu N, Crisp J. Enhancing the resilience of nurses and midwives: pilot of a mindfulness-based program for increased health, sense of coherence and decreased depression, anxiety and stress. Contemp Nurse. 2013;45(1):114-25, https://doi.org/10.5172/conu. 2013.45.1.114.

33. Goodman MJ, Schorling JB. A mindfulness course decreases burnout and improves well-being among healthcare providers. Int J Psychiatry Med. 2012;43(2):119-28, https://doi. org/10.2190/PM.43.2.b.

34. Gupta SJ, Kemper KJ, Lynn J. Interest in online interprofessional elective mind-body skills (MBS) training. Complement Ther Med. 2018;36:137-41, https://doi.org/10.1016/j.ctim. 2017.12.009.

35. Dehning S, Gasperi S, Krause D, Meyer S, Reiß E, Burger M, et al. Emotional and cognitive empathy in first-year medical students. ISRN Psychiatry. 2013;2013:801530, https:// doi.org/10.1155/2013/801530.

36. Dyrbye LN, Thomas MR, Massie FS, Power DV, Eacker A, Harper W, et al. Burnout and suicidal ideation among U.S. medical students. Ann Intern Med. 2008;149(5):334-41, https://doi.org/10.7326/0003-4819-149-5-200809020-00008.

37. Kreitzer MJ, Klatt M. Educational innovations to foster resilience in the health professions. Med Teach. 2017;39(2):1539, https://doi.org/10.1080/0142159X.2016.1248917.

38. Saunders PA, Tractenberg RE, Chaterji R, Amri H, Harazduk N, Gordon JS, et al. Promoting self-awareness and reflection through an experiential mind-body skills course for first year medical students. Med Teach. 2007;29(8):778-84, https://doi.org/10.1080/01421590701509647.

39. Dobkin PL, Laliberté V. Being a mindful clinical teacher: can mindfulness enhance education in a clinical setting? Med Teach. 2014;36(4):347-52, https://doi.org/10.3109/0142159X. 2014.887834 .

40. Malpass A, Binnie K, Robson L. Medical Students' Experience of Mindfulness Training in the UK: well-being, coping, reserve and professional development. Edu Res Int. 2019;2019:4021729, https://doi.org/10.1155/2019/4021729.

41. McConville J, McAleer R, Hahne A. Mindfulness Training for Health Profession Students-The Effect of Mindfulness Training on Psychological Well-Being, Learning and Clinical Performance of Health Professional Students: A Systematic Review of Randomized and Non-randomized Controlled Trials. Explore (NY). 2017;13(1):26-45, https://doi. org/10.1016/j.explore.2016.10.002.

42. Bond AR, Mason HF, Lemaster CM, Shaw SE, Mullin CS, Holick EA, et al. Embodied health: the effects of a mindbody course for medical students. Med Educ Online. 2013;18:1-8, https://doi.org/10.3402/meo.v18i0.20699.

43. Rosenzweig S, Reibel DK, Greeson JM, Brainard GC, Hojat M. Mindfulness-based stress reduction lowers psychological distress in medical students. Teach Learn Med. 2003;15(2):88-92, https://doi.org/10.1207/S15328015TL M1502_03.

44. Shapiro SL, Schwartz GE, Bonner G. Effects of mindfulness-based stress reduction on medical and premedical students. J Behav Med. 1998;21(6):581-99, https://doi. org/10.1023/a:1018700829825.

45. Solhaug I, Eriksen TE, de Vibe H, Haavind H, Friborg O, Sørlie T, et al. Medical and Psychology Student's Experiences in Learning Mindfulness: Benefits, Paradoxes, and Pitfalls. Mindfulness (N Y). 2016;7:838-50, https://doi.org/10.1007/ s12671-016-0521-0.

46. Turakitwanakan W, Mekseepralard C, Busarakumtragul P. Effects of mindfulness meditation on serum cortisol of medical students. J Med Assoc Thai. 2013;96 Suppl 1:S90-5.

47. Chen AK, Kumar A, Haramati A. The effect of Mind Body Medicine course on medical student empathy: a pilot study. Med Educ Online. 2016;21:31196, https://doi.org/10.3402/ meo.v21.31196.

48. Plummer C, Cloyd E, Doersam JK, Dietrich MS, Hande KA. Mindfulness in a Graduate Nursing Curriculum: A Randomized Controlled Study. Holist Nurs Pract. 2018;32(4):189-95, https://doi.org/10.1097/HNP.0000000000000277. 
49. Van Dijk I, Lucassen PL, Speckens AE. Mindfulness training for medical students in their clinical clerkships: two cross-sectional studies exploring interest and participation. BMC Med Educ. 2015;15:24, https://doi.org/10.1186/s12909-015-0302-9.

50. Barbosa P, Raymond G, Zlotnick C, Wilk J, Toomey 3rd R, Mitchell 3rd. J. Mindfulness-based stress reduction training is associated with greater empathy and reduced anxiety for graduate healthcare students. Educ Health (Abingdon). 2013;26(1):9-14, https://doi.org/10.4103/1357-6283.112794.

51. Van Vliet M, Jong MC, Jong M. A Mind-Body Skills Course Among Nursing and Medical Students: A Pathway for an Improved Perception of Self and the Surrounding World. Glob Qual Nurs Res. 2018;5:2333393618805340, https://doi. org $/ 10.1177 / 2333393618805340$.

52. Vibe Md, Solhaug I, Tyssen R, Friborg O, Rosenvinge JH, Sørlie T, et al. Mindfulness training for stress management: a randomised controlled study of medical and psychology students. BMC Med Educ. 2013;13:107, https://doi. org/10.1186/1472-6920-13-107.

53. Vibe Md, Solhaug I, Tyssen R, Friborg O, Rosenvinge JH, Sørlie T, et al. Does Personality Moderate the Effects of Mindfulness Training for Medical and Psychology Students? Mindfulness (NY). 2015;6(2):281-9, https://doi.org/10.1007/ s12671-013-0258-y.

54. Nayyar N, Saggio G, Plummer M, Jung MK, Kappenberg J. Association of Mindfulness With Residency Preference and Curriculum Selection in Preclinical Osteopathic Medical Students. J Am Osteopath Assoc. 2018;118(9):587-95, https://doi.org/10.7556/jaoa.2018.142.

55. Chung AS, Felber R, Han E, Mathew T, Rebillot K, Likourezos A. A Targeted Mindfulness Curriculum for Medical Students During Their Emergency Medicine Clerkship Experience. West J Emerg Med. 2018;19(4):762-6, https://doi. org/10.5811/westjem.2018.4.37018.
56. Dyrbye LN, Shanafelt TD, Werner L, Sood A, Satele D, Wolanskyj AP. The Impact of a Required Longitudinal Stress Management and Resilience Training Course for First-Year Medical Students. J Gen Intern Med. 2017;32(12):1309-14, https://doi.org/10.1007/s11606-017-4171-2.

57. Kuhlmann SM, Huss M, Bürger A, Hammerle F. Coping with stress in medical students: results of a randomized controlled trial using a mindfulness-based stress prevention training (MediMind) in Germany. BMC Med Educ. 2016;16(1):316, https://doi.org/10.1186/s12909-016-0833-8.

58. Vibe Md, Solhaug I, Rosenvinge JH, Tyssen R, Hanley A, Garland E. Six-year positive effects of a mindfulness-based intervention on mindfulness, coping and well-being in medical and psychology students; Results from a randomized controlled trial. PLoS One. 2018;13(4):e0196053, https://doi. org/10.1371/journal.pone.0196053.

59. Phang CK, Mukhtar F, Ibrahim N, Keng SL, Mohd Sidik S. Effects of a brief mindfulness-based intervention program for stress management among medical students: the Mindful-Gym randomized controlled study. Adv Health Sci Educ Theory Pract. 2015;20(5):1115-34, https://doi.org/10.1007/ s10459-015-9591-3.

60. Gordon JS. Mind-body skills groups for medical students: reducing stress, enhancing commitment, and promoting patient-centered care. BMC Med Educ. 2014;14:198, https:// doi.org/10.1186/1472-6920-14-198.

61. National Institutes of Health, United States National Library of Medicine Clinical Trials [Internet]. Bethesda: The Organization; 2018 [cited 2020 Jul 1]. Mindfulness and Executive Functions for Prediction of Non-technical Skills of Students in Pediatric Medical Simulations (MindExSim). Clinical Trial ID: NCT03761355 (N/ST/ZB/18/002/1206); 2018. Available from: https://clinicaltrials.gov/ct2/show/NCT 03761355 .

This work is available in Open Access model and licensed under a Creative Commons Attribution-NonCommercial 3.0 Poland License - http://creativecommons.org/ licenses/by-nc/3.0/pl/deed.en. 